\title{
Impact of Secondary Users' Field Size on Spectrum Sharing Opportunities
}

\author{
Muhammad Aljuaid \\ Department of Systems and Computer Engineering \\ Carleton University \\ Ottawa, Canada \\ Email: majuaid@sce.carleton.ca
}

\author{
Halim Yanikomeroglu \\ Department of Systems and Computer Engineering \\ Carleton University \\ Ottawa, Canada \\ Email: halim@sce.carleton.ca
}

\begin{abstract}
Previous works studied the effect of many system parameters on spectrum sharing opportunities where secondary users access the spectrum of primary users. However, a parameter that has received little attention is the spatial size of the field of secondary users. Usually, the field size is assumed to be infinite. Using results developed for infinite fields might be too pessimistic leading to missing spectrum sharing opportunities. This paper studies the effect of the field size on spectrum sharing opportunities. We verify that asymptotic results obtained for infinite fields are applicable for finite but relatively large fields as well, i.e., when the radial depth of the field is much greater than the minimum distance to the primary user. We demonstrate that in some cases, however, asymptotic results are too pessimistic hiding some spectrum sharing opportunities. Moreover, the paper shows that in certain situations a small reduction in the field size may create spectrum sharing opportunities while in certain other situations a huge increase in the field size may not eliminate spectrum sharing opportunities. Our results also suggest the possibility of a secondary network to concurrently share the spectrum with a primary user without the need for spectrum sensing techniques or other cognitive radio functionalities.

Index Terms-Spectrum Sharing, Aggregate Interference, Interference Probability.
\end{abstract}

\section{INTRODUCTION}

FCC proposal on spectrum sharing [1] has stimulated significant interest in the academia and industry due to its potential in reducing the effect of radio spectrum scarcity. In the spectrum sharing proposal, a secondary user (likely an unlicensed user) could share the spectrum with a primary user (licensed user) provided that the operation of the secondary user does not introduce "harmful interference" towards the primary user [1]. The secondary user could be a single user or a wireless network consisting of many users seen by the primary user as a field of interferers.

Some metrics have been proposed in the literature to identify whether the interference generated by secondary users reaches to a level of being "harmful" to the primary users. Examples of these metrics include the following: interference temperature [2], outage probability [3], interference indicator [4], spectral outage probability [5], and interference probability [6].

The works such as [3]-[6] study the effect of various system parameters on the harmful interference metric. However, a system parameter that has not received much attention is the

This work was funded by Saudi Aramco, Dhahran, Saudi Arabia. spatial size of the field of secondary users. In most studies, the spatial size of the field is assumed to be infinite (for brevity, the "spatial size of the field" will be simply called "field size" in the rest of the paper.)

One might argue that asymptotic results obtained for infinite fields may be used as bounds for finite fields. However, since the spectrum sharing is opportunistic, using results developed for an infinite field may end up being too pessimistic leading to missing spectrum sharing opportunities. This concern about the applicability of the results of infinite fields could be addressed properly by studying the behavior of the harmful interference metric and, hence, the spectrum sharing opportunities, with respect to the changes in the field size.

In this paper, we consider a two-dimensional field but we do not assume that the field is infinite; rather we consider a general setup in which the infinite field is a special case. We use the interference probability as the harmful interference metric. We study how the interference probability and, correspondingly, the spectrum sharing opportunities, would change with changes in the field size. The study shows that in some cases an increase in the field size may eliminate spectrum sharing opportunities; however, our study also demonstrates that there are other cases where the spectrum sharing opportunities are not affected by the increase in the field size even if the field size grows to infinity. Moreover, it is observed that while the reduction in the field size may open spectrum sharing opportunities in some cases; it does not in some other cases. To complete the picture, the effect of the field location (with respect to the primary user) on the interference probability and spectrum sharing is also discussed.

Following is an example of an immediate application of the results obtained in this paper. Currently, Wireless Sensor Networks (WSNs) are operating in unlicensed bands, e.g., $2.4 \mathrm{GHz}$. These bands are becoming over-crowded which may severely affect the operation of WSNs. Alternatively, the WSNs could share a licensed band with a primary user. However, this sharing usually requires spectrum sensing and cognitive radio functionalities. Adding these functionalities may increase the complexity of the sensor nodes to a level that is economically infeasible with today's technologies especially for massive deployments [7]. It has been shown in this paper that under certain conditions secondary users (WSNs 
in this example) could concurrently and continuously share the spectrum with a primary user without the need to have any spectrum sensing techniques or cognitive radio functionalities.

The rest of the paper is organized as follows. First, a description of the system model used in this paper is given in Section II. Then, Section III focuses on the formulation of the mean of the aggregate interference, and discusses its behavior with respect to changes in the field size and location. The variance of the aggregate interference is studied in Section IV. The upper bound on the interference probability is covered in Section V. The effect of the field size and location on the spectrum sharing opportunities is discussed in Section VI. Finally, Section VII summarizes the main results in this paper.

\section{SYSTEM MODEL}

In this paper, we consider a field of secondary users with an annular sector shape as shown in Fig. 1. The total area of this field is denoted by $A$. The shortest distance between the field and the victim (i.e., primary user) receiver ${ }^{1}$ is shown by $r_{o}$. The radial depth of the field is represented by $L$. The field spans over an angle of $\theta$ as seen by the primary user. This model is general enough to encompass many different scenarios, e.g., a finite secondary field, an infinite secondary field, a primary user away from the secondary field, and a primary user in the middle of the secondary field.

The aggregate interference of this field as seen by the primary user is denoted by $I_{A}$ which can be expressed as

$$
I_{A}=\sum_{i \in \aleph} I_{i}
$$

where $\aleph$ is the set of concurrently transmitting interfering nodes. These interfering nodes are assumed to be spatially distributed according to a Poisson Point Process (PPP) [8]. Each node is introducing an instantaneous interference power of $I_{i}$ at the primary user. The cardinality of $\aleph$ is $N$ which is a random variable following a Poisson distribution with an average value of $D A$ where $D$ is the average number of transmitting nodes in a unit area. $I_{i}$ 's are assumed to be i.i.d. random variables. By considering the commonly used path loss model, $I_{i}$ can be expressed as

$$
I_{i}=W_{i} r_{i}^{-n},
$$

where $r_{i}$ is the distance between node $i$ and the primary user and it is restricted to be greater than or equal to $1 \mathrm{~m}$ for the model in (2) to be valid. Allowing $r_{i}$ to be less than $1 \mathrm{~m}$ will lead to singularity at $r_{i}=0[6]$. The parameter $n(>2)$ is the path loss exponent, and $W_{i}$ is a random variable that results from the multiplications of the other system and propagation parameters such as transmitter power, antenna gains, and the random factors capturing the effect of shadowing and possibly multipath fading. $W_{i}$ and $r_{i}$ are assumed to be independent random variables.

\footnotetext{
${ }^{1}$ While the analysis in this paper focuses on a single primary user, it can be used directly or with some extensions to investigate the effect of a secondary field on a primary field (consisting of many primary users).
}

An indicator variable $B_{i}$ can be introduced in the formulation in order to capture the effect of the overlap of the frequency bands used by an interfering node $i$ and the primary user. This indicator has a range that goes from 0 to 1 ; it is equal to 1 if the frequency band of the interfering node is totally within the frequency band of the primary user, whereas the indicator is equal to zero if the frequency band of the interference is outside the frequency band of the primary user and does not cause an adjacent channel interference. In this paper, $B_{i}$ is considered to be 1 which corresponds to the worst case. The results reported in this paper can easily be extended to consider the cases where $B_{i}<1$. This extension can be facilitated by multiplying the right side in (2) by $B_{i}$.

The harmful interference metric that is used in this paper is the interference probability. This metric can be described as

$$
P\left(I_{A} \geq I_{t h}\right) \leq \beta,
$$

which means that the probability of the aggregate interference being greater than a certain interference threshold, $I_{t h}$, should not exceed $\beta$, where $\beta \ll 1$ [6]. If (3) is not violated, then the aggregate interference is considered to be non-harmful.

We are interested in studying how the interference probability and, hence, the spectrum sharing behave with respect to changes in the field size, mainly $L$, and the location of the field, i.e., changes in $r_{o}$. To achieve this, the distribution of $I_{A}$ or at least some of its characteristics are required. The characteristic function of the aggregate interference generated by a poisson field of interferer has been derived in [3], [6], [8]-[10]. While the characteristic function is known, the cumulative distribution function and probability density function have no closed-form expression except for a special case when the path loss exponent is 4 and the field is infinite with no exclusion region around the victim receiver [8]. Therefore, authors in [3] and [6] end up working with approximations, and authors in [9] use upper bounds based on Chebyshev's inequality. Similarly, in this present paper, Chebyshev's inequality is utilized to establish an upper bound on the interference probability. To use this inequality, the first two moments of $I_{A}$ have to be identified. In the following sections, formulations for these moments and the upper bound on the interference probability are developed.

\section{Mean of the Aggregate Interference}

This section addresses the formulation of the mean of the aggregate interference $\left(\mu_{A}\right)$ and discusses the behavior of this mean with respect to changes in the field size and location.

\section{A. The Formulation}

Taking the expectation of $I_{A}$ in (1) results in

$$
\mu_{A}=E\left[I_{A}\right]=E\left[\sum_{i \in \aleph} I_{i}\right] .
$$

Assuming that $I_{i}$ 's are i.i.d. random variables,

$$
\mu_{A}=E[N] E\left[I_{i}\right]=\mu_{N} \mu_{I} .
$$


Since the number of transmitting nodes in an area follows a Poisson distribution,

$$
\mu_{N}=D A
$$

Using (2) and the assumption that $W_{i}$ and $r_{i}$ are independent, $\mu_{I}$ can be expressed as

$$
\mu_{I}=\mu_{W} E\left[r_{i}^{-n}\right],
$$

where $\mu_{W}=E\left[W_{i}\right]$. Since the nodes are spatially distributed according to a PPP, the locations of the nodes follow a uniform distribution over $A$ [8]. Thus,

$$
E\left[r_{i}{ }^{-n}\right]=\frac{\theta}{A(n-2)} r_{o}{ }^{2-n}\left[1-\left(1+\frac{L}{r_{o}}\right)^{2-n}\right] .
$$

From (5)-(8), $\mu_{A}$ can be obtained as

$$
\mu_{A}=\frac{D \theta}{(n-2)} r_{o}^{2-n} \mu_{W}\left[1-\left(1+\frac{L}{r_{o}}\right)^{2-n}\right] \text {. }
$$

\section{B. Effect of Field Size and Location on $\mu_{A}$}

The behavior of the mean with respect to the changes in the field size is discussed thoroughly in [11]. In brief, the mean of the aggregate interference changes linearly with changes in $L$ when $L \ll r_{o}$. For $L \gg r_{o}$, the changes in $L$ does not significantly affect the mean of the aggregate interference, rather the mean converges to a value of

$$
\mu_{A}=\frac{D \theta}{(n-2)} \mu_{W} r_{o}^{2-n} \text {. }
$$

This convergence observation agrees with the findings reported in [8] and [12] which indicate that the aggregate interference power generated by an infinite field of interferers converges if the path loss exponent is greater than the dimensions of the field ${ }^{2}$.

The effect of the field location on the mean of the aggregate interference can be summarized as follows: a $10 \mathrm{~dB}$ increase in $r_{o}$ leads to a $10(n-2) \mathrm{dB}$ decrease in $\mu_{A}$ for $r_{o} \ll L$; when $r_{o} \gg L$, a $10 \mathrm{~dB}$ increase in $r_{o}$ results in $10(n-1) \mathrm{dB}$ decrease in the mean of the aggregate interference.

\section{VARIANCE OF THE Aggregate INTERFERENCE}

The variance of the aggregate interference of the field of secondary users is denoted by $\sigma_{A}^{2}$. This section presents the formulation of $\sigma_{A}^{2}$ and discusses its behavior with respect to changes in $L$ and $r_{o}$.

\section{A. The Formulation}

Based on the assumptions that all $I_{i}$ 's are i.i.d. random variables, it can be shown that the second moment of the aggregate interference for $N$ transmitting nodes is

$$
E\left[I_{A}^{2} \mid N\right]=N\left(\sigma_{I}^{2}+\mu_{I}^{2}\right)+N(N-1) \mu_{I}^{2} .
$$

Averaging (11) over $N$ results in

$$
\sigma_{A}^{2}=\mu_{N} \sigma_{I}^{2}+\sigma_{N}^{2} \mu_{I}^{2} .
$$

\footnotetext{
${ }^{2}$ The divergence of the aggregate interference power for an infinite twodimensional field is discussed in [11], [13], [14].
}

Since $N$ follows a Poisson distribution,

$$
\sigma_{N}^{2}=\mu_{N}=D A \text {. }
$$

By noting that

$$
\begin{gathered}
\sigma_{I}^{2}=E\left[W^{2}\right] E\left[r_{i}^{-2 n}\right]-\mu_{I}^{2}, \\
E\left[r_{i}^{-2 n}\right]=\frac{\theta r_{o}^{2-2 n}}{A(2 n-2)}\left[1-\left(1+\frac{L}{r_{o}}\right)^{2-2 n}\right]
\end{gathered}
$$

and substituting them as well as (13) in (12), we obtain

$$
\sigma_{A}^{2}=\frac{D \theta r_{o}^{2-2 n}}{2 n-2} \mu_{W}^{2}\left(1+\frac{\sigma_{W}^{2}}{\mu_{W}^{2}}\right)\left[1-\left(1+\frac{L}{r_{o}}\right)^{2-2 n}\right],
$$

which means that the randomness in $W_{i}$ causes an increase by a factor of $\left(1+\frac{\sigma_{W}^{2}}{\mu_{W}^{2}}\right)$. So, the behavior of the variance of the aggregate interference with respect to changes in the field size and location can be studied without loss of generality by assuming that $W_{i}$ is deterministic.

\section{B. Effect of Field Size and Location on $\sigma_{A}^{2}$}

The variance of the aggregate interference changes linearly with changes in $L$ when $L \ll r_{o}$. However, when $L \gg r_{o}$ the changes in $L$ has no tangible effect on the variance. The variance of the aggregate interference saturates with respect to the increase in $L$ at

$$
\sigma_{A}^{2}=\frac{D \theta r_{o}^{2-2 n}}{2 n-2} \mu_{W}^{2}\left(1+\frac{\sigma_{W}^{2}}{\mu_{W}^{2}}\right) .
$$

Regarding the effect of the field location on the variance of the aggregate interference, a $10 \mathrm{~dB}$ increase in $r_{o}$ leads to $10(2 n-2) \mathrm{dB}$ decrease in the variance when $r_{o} \ll L$, and a $10(2 n-1) \mathrm{dB}$ decrease if $r_{o} \gg L$.

\section{UPPER BOUND ON THE INTERFERENCE PROBABILITY}

\section{A. The Formulation}

An upper bound on the interference probability can be established using Chebyshev's inequality ${ }^{3}$. This inequality states that for a random variable $X$ with mean $\mu$ and variance $\sigma^{2}[15]$

$$
P(|X-\mu| \geq t) \leq \frac{\sigma^{2}}{t^{2}}, t>0 .
$$

Applying this inequality to our problem results in

$$
P\left(I_{A} \geq I_{t h}\right) \leq \frac{\frac{D \theta r_{o}^{2-2 n}}{2 n-2} \mu_{W}^{2}\left(1+\frac{\sigma_{W}^{2}}{\mu_{W}^{2}}\right)\left[1-\left(1+\frac{L}{r_{o}}\right)^{2-2 n}\right]}{\left(I_{t h}-\frac{D \theta r_{o}^{2-n}}{(n-2)} \mu_{W}\left[1-\left(1+\frac{L}{r_{o}}\right)^{2-n}\right]\right)^{2}}
$$

provided that $I_{t h}>\mu_{A}$ where $I_{t h}$ could be the maximum level of the tolerable interference by the primary user.

The expression (19) can be used to understand the effect of the randomness in $W_{i}$ on the probability of interference. As mentioned before, the randomness in $W_{i}$ may be due to the

${ }^{3}$ While Chebyshev's inequality may result in loose upper bounds, the
decisions deduced from these bounds are conservative and on the favor of protecting the primary user. 
fluctuations in the transmitted power, multipath fading, and more importantly, the shadowing effect. The randomness in $W_{i}$ shifts up the upper bound of the interference probability by $10 \log \left(1+\frac{\sigma_{W}^{2}}{\mu_{W}^{2}}\right) \mathrm{dB}$. So, without loss of generality, the behavior of the upper bound on the interference probability with respect to changes in field size and location can be studied with the assumption that $W_{i}$ is deterministic. The results could be adjusted with a proper scaling factor (or a proper $\mathrm{dB}$ shift) to reflect the randomness in $W_{i}$.

\section{B. Effect of Field Size and Location on the Interference Probability}

Before discussing the effect of changes in the field size and location on the interference probability, it is important to highlight the following: the Chebyshev inequality is valid when $I_{t h}>\mu_{A}$; however, $\mu_{A}$ would change with changes in the field size and location. Therefore, in our plots we make sure that $I_{t h}>\mu_{A}$ over the ranges of $L$ or $r_{o}$ considered in the plots. In practice, the value of $I_{t h}$ is determined by the regulator or the primary user, as such, the secondary user has no control on it.

The behavior of the upper bound on the interference probability with respect to the changes in the field size is shown in Fig. 2. The upper bound on the interference probability increases linearly with the increase in $L$ for $L \ll r_{o}$. If $L \gg r_{o}$, then the increase in $L$ has no or negligible effect on the upper bound. In this case, the upper bound saturates at

$$
P\left(I_{A} \geq I_{t h}\right) \leq \frac{\frac{D \theta}{2 n-2} r_{o}^{2-2 n} \mu_{W}^{2}\left(1+\frac{\sigma_{W}^{2}}{\mu_{W}^{2}}\right)}{\left(I_{t h}-\frac{D \theta}{n-2} r_{o}^{2-n} \mu_{W}\right)^{2}} .
$$

Figure 3 shows the behavior of the upper bound on the interference probability with respect to changes in $r_{o}$. A 10 $\mathrm{dB}$ increase in $r_{o}$ leads to a $10(2 n-2) \mathrm{dB}$ decrease in the upper bound if $r_{o} \ll L$; when $r_{o} \gg L$, a $10 \mathrm{~dB}$ increase in $r_{o}$ leads to a $10(2 n-1) \mathrm{dB}$ decrease in the upper bound.

\section{EfFect of Field Size and Location on Spectrum SHARING OPPORTUNITIES}

We consider a spectrum sharing opportunity exists if the condition in (3) is satisfied. Therefore, for a certain value of $I_{t h}$, the upper bound obtained in (19) will be compared to (3). If the upper bound is less than or equal to $\beta$, then the aggregate interference of the secondary network is considered to be non-harmful to the primary user (non-interfering region). Otherwise, the secondary network may cause harmful interference to the primary user (interfering region).

If the secondary network operates in an interfering region, then it may use the spectrum of the primary user during the absence of the primary user. However, when the primary user starts using the spectrum, then the secondary user has to evacuate the spectrum or to adapt its transmission parameters to move to the non-interfering region. Therefore, the secondary user has to have a technology such as "cognitive radio" to be able to sense the presence of the primary user, and to perform a proper action accordingly [16], [17].
On the other hand, if the secondary network operates in the non-interfering region, then it can concurrently and continuously share the spectrum with the primary user without the need to implement any spectrum sensing technique or cognition related function. This result could be used towards solving the spectrum problem facing WSNs without increasing the complexity of the sensor nodes. Currently, WSNs are operating in unlicensed bands which are becoming more and more crowded; this may severely affect the operation of WSNs. Based on the result mentioned above, the WSNs could alternatively share a licensed band with a primary user without the need to have sophisticated functionalities like those in cognitive radios.

The effect of the field size and location on determining the interfering and non-interfering regions and on the spectrum sharing opportunities are discussed in the following subsections. Without loss of generality, the numerical examples and results shown later in the paper are based on the following model for $W_{i}$ :

$$
W_{i}=P_{i} G_{i} G_{x}\left(\frac{\lambda}{4 \pi}\right)^{2} d_{o}^{n-2} h_{i} 10^{\frac{S_{i}}{10}},
$$

where $P_{i}$ is the transmitted power by node $i, G_{i}$ is its antenna gain, $G_{x}$ is the antenna gain of the primary user, $\lambda$ is the wavelength of the carrier frequency, $d_{o}$ is the close-in reference distance, $h_{i}$ reflects the effect of the multipath fading on the received power, and $S_{i}$ is a Gaussian random variable modeling the shadowing effect.

\section{A. Field Size and Spectrum Sharing Opportunities}

The discussion in this subsection is based on Fig. 4. This figure has four curves; each curve corresponds to a certain value of $\beta$. Each one of these curves divides the figure into two main regions: interfering (comprised of zones 1 and 2) and non-interfering (comprised of zones 3 and 4) regions.

If the maximum interference probability that a primary user can tolerate is $\beta$ (e.g., 0.01), then the aggregate interference generated by the secondary network is not considered to be harmful if the interference probability is less than $\beta$. Therefore, the part of Fig. 4 above the curve of $\beta=0.01$ is considered to be a non-interfering region. In this case, a secondary network could concurrently and continuously share the spectrum with the primary user without the need to have spectrum sensing techniques or cognitive radio capabilities.

The non-interfering region in Fig. 4 can be divided into two zones: zone 3 and zone 4 . In zone 4 , the secondary network does not cause harmful interference towards the primary users regardless of the field size, $L$. Therefore, expanding the field does not eliminate any spectrum sharing opportunity. In zone 3 , on the other hand, the increase in $L$ may move the secondary network from the non-interfering region to the interfering region eliminating a spectrum sharing opportunity.

For a specific $\beta$ value, the part of Fig. 4 below the curve corresponding to that $\beta$ (e.g., 0.01 ) is considered to be an interfering region because the interference probability in this part is grater than 0.01 . The interfering region can also be 
divided into two zones. In the first one (zone 1), a reduction in $L$ never moves the secondary network from the interfering region to the non-interfering region except when there is no transmitting node. Therefore, controlling the field size here does not create a spectrum sharing opportunity. In the other zone (zone 2), on the other hand, a reduction in $L$ may lead to moving the secondary network from the interfering region to the non-interfering region which creates a spectrum sharing opportunity.

At this stage we can comment on the impact of the field size on the spectrum sharing opportunities. It has been shown that an increase in the field size may eliminate spectrum sharing opportunities. However, there are some cases where the spectrum sharing opportunities are not affected by the increase in the field size even if the field size grows to infinity. Moreover, it has been demonstrated that the reduction in the field size may open spectrum sharing opportunities. On the other hand, there are some other cases where the reduction in the field size does not open any spectrum sharing opportunities.

Regarding the applicability of asymptotic results obtained for a field of an infinite size to the case where the field size is finite, we can state the followings: asymptotic results obtained for infinite fields can be applied for finite fields whose radial depth $(L)$ is much greater than the minimum distance between the field and the primary user $\left(r_{o}\right)$. Otherwise, these asymptotic results will be too conservative and may lead to missing spectrum sharing opportunities.

\section{B. Field Location and Spectrum Sharing Opportunities}

Figure 5 has four curves. Each curve corresponds to a certain value of $\beta$. Each one of these curves divides the figure into two regions: interfering and non-interfering regions.

A parameter that the secondary network may use to move from an interfering region to a non-interfering region is $r_{o}$. By knowing the maximum acceptable interference probability $\beta$ in (3) and $I_{t h}$, Fig. 5 can be used to find the minimum exclusion region $\left(r_{o}\right)$ satisfying these requirements. For example, if $I_{t h}=-80 \mathrm{dBm}$, then $r_{o}$ should be greater than or equal to $630 \mathrm{~m}$ to ensure that the interference probability is less than or equal to 0.1 .

The value of $r_{o}$ can be properly designed during the predeployment stages of the secondary network. For an already deployed secondary network, it is possible to virtually control $r_{o}$ by a MAC protocol forcing nodes within a distance of $r_{o}$ from the primary user not to transmit.

Figure 5 also reveals an interesting situation. The right side of the plot where the lines of different $\beta$ 's overlap may suggest that for sufficiently high $r_{o}$ there is no need to know the distribution of the aggregate interference; knowing only the mean is sufficient. In other words, the aggregate interference in this case is tending towards a deterministic quantity. Similar observations is made in [10] while discussing the ratio of the standard deviation of the aggregate interference to its mean.

\section{CONCLUSIONS}

This paper studies the impact of the field size of secondary users on spectrum sharing opportunities. The study shows that an increase in the field size may eliminate the spectrum sharing opportunities. However, there are some cases where the spectrum sharing opportunities are not reduced by the increase in the field size even when the field size grows to infinity.

The paper demonstrates that asymptotic results obtained for an infinite field could be applied for a finite field whose radial depth is much greater than the minimum distance between the field and the primary user. Otherwise, these asymptotic results will be too conservative and may lead to missing spectrum sharing opportunities.

The results in this paper show the possibility of a secondary network to concurrently and continuously share the spectrum with a primary network without the need for spectrum sensing techniques or other cognitive radio functionalities. This observation hints a promising spectrum sharing solution for the wireless sensor networks which are facing interference challenges in the crowded unlicensed spectrum.

\section{REFERENCES}

[1] "Spectrum policy task force," Federal Communications Commission (FCC), ET Docket no. 02-135, Nov. 2002.

[2] P. Kolodzy, "Interference temperature: a metric for dynamic spectrum utilization," Int. J. Network Mgmt, vol. 16, no. 2, pp. 103-113, Mar. 2006.

[3] R. Menon, R. M. Buehrer, and J. H. Reed, "Outage probability based comparison of underlay and overlay spectrum sharing techniques," in Proc. 1st IEEE Symp. Dynamic Spectrum Access Networks (DySPAN'05), Baltimore, MD, USA, Nov. 2005, pp. 101-109.

[4] "Spectrum commons classes for license-exemption," Office of Communications (OFCOM), Tech. Rep., Nov. 2008.

[5] P. C. Pinto and M. Z. Win, "Spectral characterization of wireless networks," IEEE Wireless Commun. Mag., vol. 14, no. 6, pp. 27-31, Dec. 2007.

[6] A. Ghasemi and E. S. Sousa, "Interference aggregation in spectrumsensing cognitive wireless networks," IEEE J. Sel. Topics Signal Process., vol. 2, no. 1, pp. 41-56, Feb. 2008.

[7] F. Granelli, H. Zhang, X. Zhou, and S. Maranò, "Research advances in cognitive ultra wide band radio and their application to sensor networks," Mobile Networks and Applications, vol. 11, no. 4, pp. 487-499, Aug. 2006.

[8] E. S. Sousa and J. A. Silvester, "Optimum transmission ranges in a direct-sequence spread-spectrum multihop packet radio network," IEEE J. Sel. Areas Commun., vol. 8, no. 5, pp. 762-771, June 1990.

[9] S. Weber, J. Andrews, and N. Jindal, "The effect of fading, channel inversion, and threshold scheduling on ad hoc networks," IEEE Trans. Inf. Theory, vol. 53, no. 11, pp. 4127-4149, Nov. 2007.

[10] R. Etkin, "Spectrum sharing: fundamental limits, scaling laws, and selfenforcing protocols," Ph.D. dissertation, EECS Department, University of California, Berkeley, CA, USA, 2006.

[11] M. Aljuaid and H. Yanikomeroglu, "On the asymptotic analysis of average interference power generated by a wireless sensor network," in Proc. IEEE VTC2008-Fall, Calgary, AB, Canada, Sep. 2008.

[12] M. Haenggi, "On distances in uniformly random networks," IEEE Trans. Inf. Theory, vol. 51, no. 10, pp. 3584-3586, Oct. 2005.

[13] R. Mathar and J. Mattfeldt, "On the distribution of cumulated interference power in rayleigh fading channels," Wireless Networks, vol. 1, no. 1, pp. 31-36, Feb.-Mar. 1995.

[14] T. J. Shepard, "A channel access scheme for large dense packet radio networks," in Proc. of ACM SIGCOMM'96, Stanford, CA, USA, Aug. 1996. [Online]. Available: http://www.acm.org/sigcomm/sigcomm96/ papers/shepard.ps 


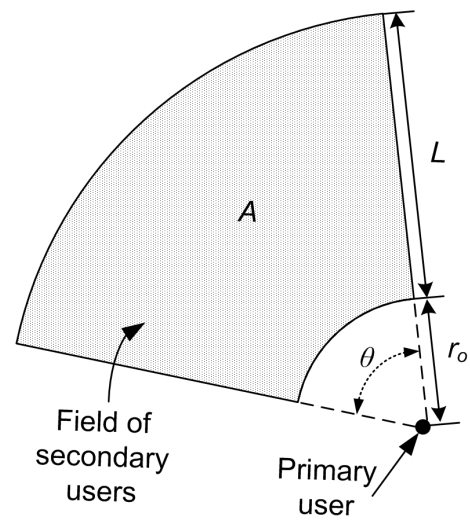

Fig. 1. Field layout.

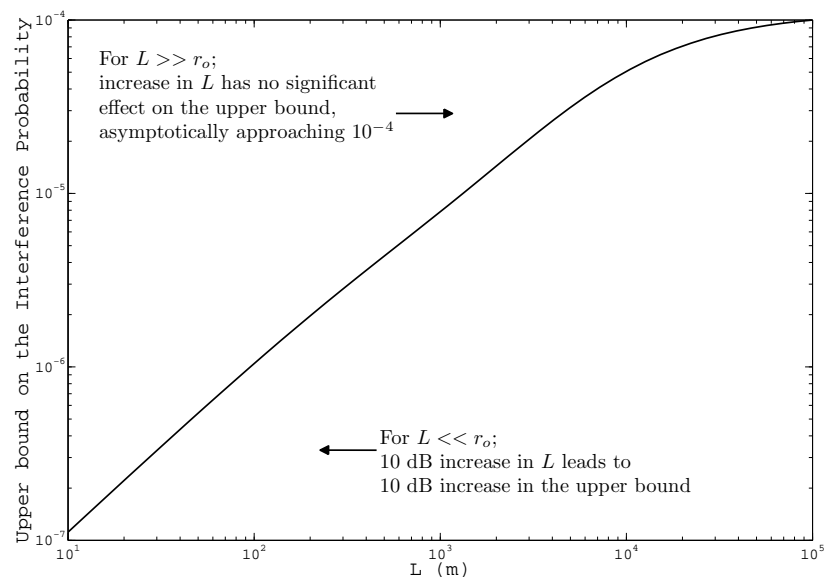

Fig. 2. Effect of field size on interference probability $\left(r_{o}=1000 \mathrm{~m}\right.$; see Table I for other assumptions used to produce this plot).

TABLE I

System and Propagation Parameters Used to Produce Plots

\begin{tabular}{c|c|l}
\hline Parameter & Value & Remarks \\
\hline$n$ & 3 & \\
$P_{i}$ & $1 \mathrm{~mW}$ & Omni antenna \\
$G_{i}, G_{x}$ & $0 \mathrm{dBi}$ & \\
$f$ & $2.4 \mathrm{GHz}$ & \\
$d_{o}$ & $1 \mathrm{~m}$ & \\
$\theta$ & $2 \pi$ & \\
$D$ & 0.01 node $/ \mathrm{m}^{2}$ & \\
$\sigma_{W}^{2}$ & $W_{i}$ assumed to be deterministic & \\
\hline
\end{tabular}

[15] W. Feller, An Introduction to Probability Theory and Its Applications, 2nd ed. New York: Wiley, 1971.

[16] S. Haykin, "Cognitive radio: brain-empowered wireless communications," IEEE J. Sel. Areas Commun., vol. 23, no. 2, pp. 201-220, Feb. 2005.

[17] I. Akyildiz, W. T. Lee, M. Vuran, and S. Mohanty, "Next generation/dynamic spectrum access/cognitive radio wireless networks: a survey," Comp. Networks J., vol. 50, no. 13, pp. 2127-2159, Sep. 2006.

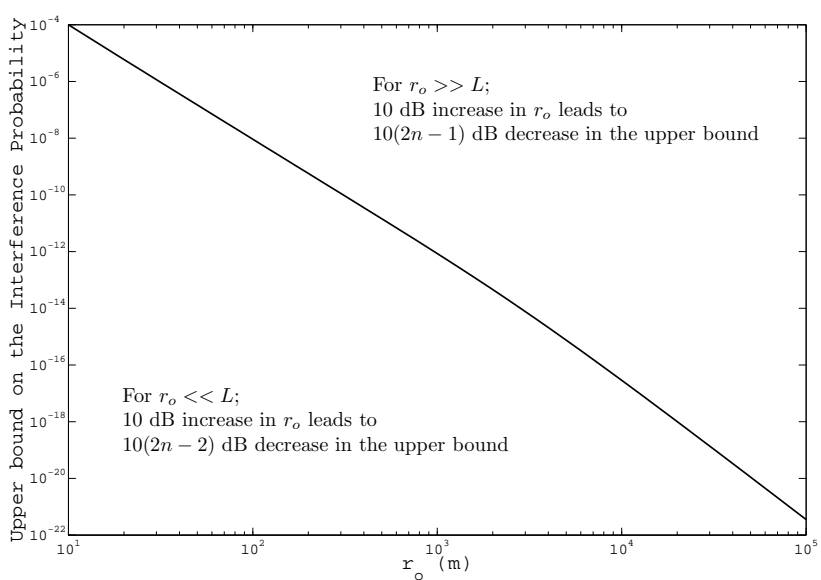

Fig. 3. Effect of field location on interference probability ( $L=1000 \mathrm{~m}$; see Table I for other assumptions used to produce this plot).

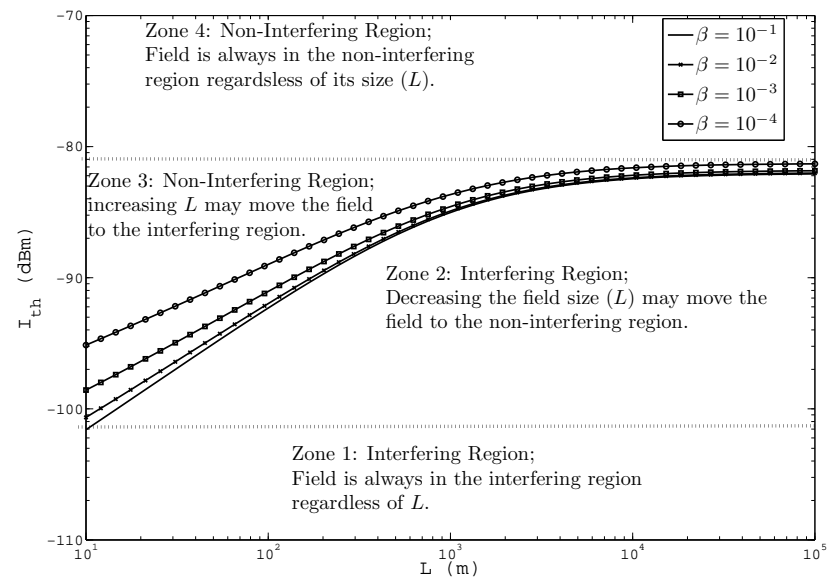

Fig. 4. Field size and spectrum sharing opportunities $\left(r_{o}=1000 \mathrm{~m}\right.$; see Table I for other assumptions used to produce this plot).

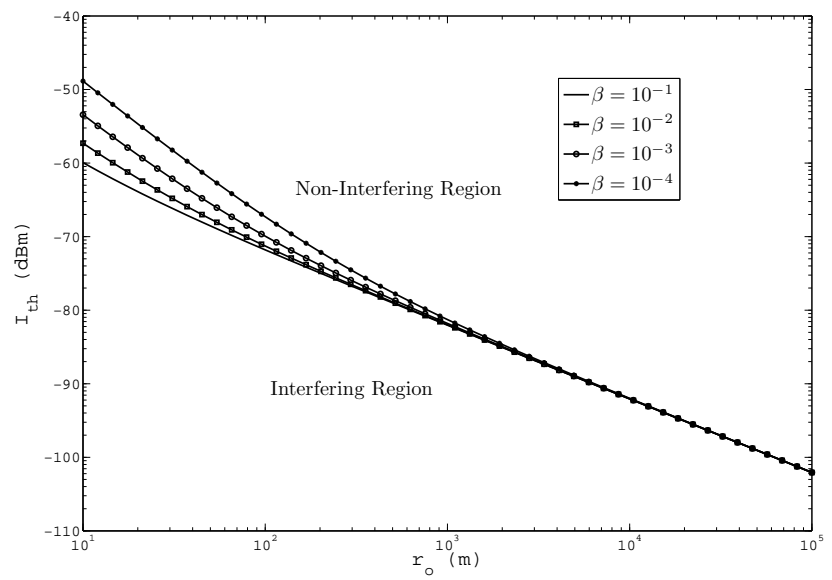

Fig. 5. Field location and spectrum sharing opportunities $(L \rightarrow \infty$; see Table I for other assumptions used to produce this plot). 